\title{
As seis fases da gestão por processos: proposta de um método
}

\begin{tabular}{|c|c|}
\hline \multirow[t]{2}{*}{ Bernardo Dias Machado } & $\begin{array}{l}\text { Universidade Federal do Rio Grande do Sul (UFRGS), Departamento de } \\
\text { Produção e Transportes, Programa de Pós-Graduação em Engenharia de } \\
\text { Produção, Porto Alegre, RS, Brasil. }\end{array}$ \\
\hline & E-mail: bernardo.machado@ufrgs.br \\
\hline \multirow[t]{2}{*}{ Jorge Estuardo Tello Gamarra } & $\begin{array}{l}\text { Universidade Federal do Rio Grande (FURG), Escola de Química e Alimentos, } \\
\text { Conselho de Ensino Pesquisa e Extensão, Santo Antônio da Patrulha, RS, } \\
\text { Brasil. }\end{array}$ \\
\hline & E-mail:jorgetellogamarra@gmail.com \\
\hline \multirow[t]{2}{*}{ Denise Lindstrom Bandeira } & $\begin{array}{l}\text { Universidade Federal do Rio Grande do Sul (UFRGS), Escola de } \\
\text { Administração, Departamento de Ciências Administrativas, Porto Alegre, RS, } \\
\text { Brasil. }\end{array}$ \\
\hline & E-mail: dlbandeira@ea.ufrgs.br \\
\hline \multirow[t]{2}{*}{ Cláudio José Muller } & $\begin{array}{l}\text { Universidade Federal do Rio Grande do Sul (UFRGS), Departamento de } \\
\text { Produção e Transportes, Programa de Pós-Graduação em Engenharia de } \\
\text { Produção, Porto Alegre, RS, Brasil. }\end{array}$ \\
\hline & E-mail: cmuller@producao.ufrgs.br \\
\hline Carla Beatriz da Luz Peralta & $\begin{array}{l}\text { Universidade Federal do Pampa (UNIPAMPA), Departamento de } \\
\text { Engenharias, Programa de graduação em Engenharia de Produção, Bagé, } \\
\text { RS, Brasil. }\end{array}$ \\
\hline
\end{tabular}

Recebido em: 23 maio 2018. Aceito em: 23 ago 2018.

DOI: http://dx.doi.org/10.21674/2448-0479.45.651-668

\section{Resumo}

Métodos voltados à gestão por processos têm sido importantes ferramentas para melhorar o desempenho operacional. Contudo, apesar dos aportes destes métodos, maiores avanços ainda são necessários. O presente artigo tem como objetivo apresentar um método para a gestão por processos, contendo seis fases: conhecimento da organização, seleção do processo, mapeamento do processo, análise do processo, estabelecimento de mudanças e implementação e monitoramento do processo. Para poder analisar a utilidade desta proposta, foi realizado um estudo de caso em uma farmácia de manipulação. Os resultados obtidos nesse estudo de caso permitiram a redução de $23 \%$ no tempo de ciclo do produto e de $30 \%$ no tempo gasto para a produção de uma unidade, resultando em um aumento de produtividade em torno de $31 \%$. No que diz respeito à empresa, a aplicação do método proporcionou evolução organizacional, pois houve a efetivação da aplicação do método no contexto da empresa, aprendizagem dos conceitos sobre a gestão por processo e por fatores como: o conhecimento por parte da empresa sobre a nova forma de gerenciamento, a conscientização por parte dos colaboradores envolvidos com o projeto e da alta direção a respeito da necessidade dessa mudança e o empenho dos mesmos na busca de informações relevantes para a tomada de decisão sobre as mudanças.

Palavras-chave: Processos. Gestão por Processos. Fases do BPM. 


\section{Abstract \\ The six stages of management by processes: proposal for a method}

Methods aimed to the management by processes have been important tools to improve the operational performance. However, despite the inputs of those methods, greater improvements are still necessary. The present article aims to submit a method for a management by processes, holding six stages: organizational knowledge, process selection, process mapping, process analysis, change establishment, and process implementation and monitoring. To be able to analyze the usefulness of that proposal, a case study was performed at a manipulation pharmacy. The results obtained in that case study allowed the decrease of $23 \%$ of the product cycle time and a decrease of $30 \%$ of the time spent on the production of one unit, resulting in an increase in productivity of around $31 \%$. With regards to the company, the application of the method provided a significant organizational evolution, since there was the establishing of the introduction, in its context, of concepts about management by processes through factors like: the company knowledge about the new method of management, the awareness of the collaborators involved with the project and awareness of the senior management regarding that need for change and their commitment to searching for relevant information for the decision-making about the changes.

Keywords: Processes. Management by Processes. Phases of BPM.

\section{Introdução}

Para a conquista de espaço em um mercado competitivo, toda empresa deve buscar meios que permitam o alcance da excelência, exigindo que as organizações façam uma reavaliação ininterrupta das tendências de mercado e de suas posturas, trazendo grandes desafios para seus administradores. De acordo com Araújo (2012), um dos caminhos plausíveis para alcançar essa excelência é o desenvolvimento de um modelo gerencial orientado a processos. Tal gestão possibilita, segundo Maranhão e Macieira (2004), que se analise a interfuncionalidade dos processos organizacionais no intuito de melhorá-los. A gestão por processos objetiva a descoberta do que é realizado pela organização e, a partir disso, desenvolve formas de otimização do trabalho (ARAÚJO, 2012; RECKER; MENDLING, 2016).

Nas últimas décadas, muitas técnicas têm sido propostas e aplicadas. Entretanto, percebeu-se, no decorrer desta pesquisa, uma oportunidade para a proposição de um método, integrando os conceitos e práticas de outras ferramentas.

O objetivo deste estudo é propor um novo método para a gestão de processos, dividido em seis fases, para busca de soluções gerenciais visando à melhoria do desempenho operacional. Para validar a pesquisa, foi analisado o processo produtivo de uma farmácia de 
manipulação, a fim de introduzir conceitos voltados a uma gestão orientada a processos visando à melhoria de sua eficiência e produtividade.

Este artigo está organizado como segue: a seção 2 apresenta a visão por processos nas organizações; a seção 3 analisa os diferentes métodos de melhoramento e gestão de processos; na seção 4 é proposto um método para o gerenciamento de processos; a seção 5 apresenta o método de pesquisa; na seção 6, é analisado o estudo de caso; e a seção 7 contém as considerações finais.

\section{Visão por processos nas organizações}

Quando trazido ao ambiente organizacional, o termo processo, conforme Guha e Kettinger (1993) e Trkman (2010), é compreendido como um conjunto coordenado de atividades, representando uma solução para a agregação de valor ou, ainda, segundo Davenport (1993), um conjunto estruturado e mensurado de atividades designado a produzir um output específico (Figura 1).

Figura 1 - Esquema de um processo

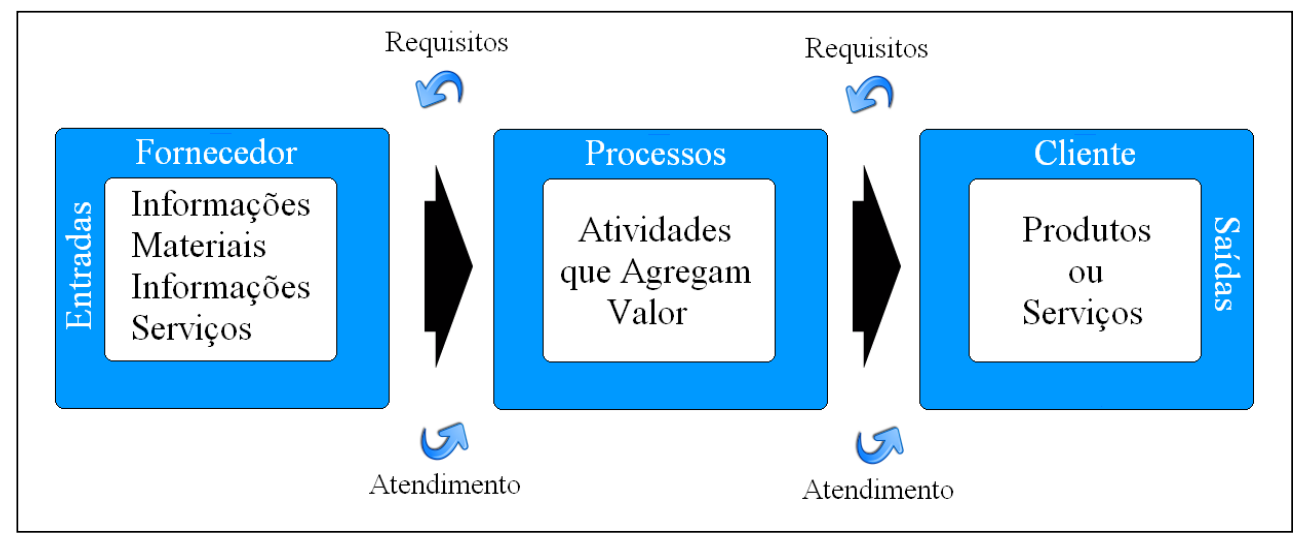

Fonte: Adaptado de Davenport (1993).

Ao longo da história, houve mudanças significativas na forma de visualizar e atender esses processos. Segundo Slack et al. (2008), foi em 1970 e 1980 que o gerenciamento de processos, com o nome de gerenciamento de operações, tornou-se popular. Porém, no final da década de 1980 surgiu um novo modelo de gestão dos processos oposto à abordagem científica de Taylor. Passou a predominar a visão sistêmica, uma visualização da empresa como um sistema inter-relacionado, objetivando a compreensão pelos departamentos de seus impactos nas demais estruturas organizacionais (SLACK et al., 2008; JACOBS et al., 2009; VAN DER AALST et al., 2016). Já no século $\mathrm{XXI}$, o sistema de gerenciamento empresarial será fundamental para o melhor desempenho organizacional. Com isso, surge a preocupação dos gestores com programas de gerenciamento como a gestão por processos, capaz de eliminar ineficiências, aumentar a satisfação dos clientes e alcançar melhores práticas (PORTER, 1996; IDEN, 2012; RECKER; MENDLING, 2016). 
Caso haja o entendimento, pelos gestores, de que os processos não são isolados, mas interdependentes, a organização estará adotando uma visão por processos e não mais por atividades (BALDAM et al., 2007). Esse fato possibilita a descoberta do que é feito pela organização de forma mais sistemática e, com isso, a descoberta de formas de otimizar o trabalho (ARAÚJO, 2012). Nas organizações tradicionais, segundo Hammer (1998), os processos são ignorados, o que não ocorre nas organizações orientadas por processos, onde são projetados e mensurados.

A partir daí, é relevante o ponto de vista de Rummler e Brache (1994) e Storch et al. (2013) a respeito da vantagem de uma visão por processos, pois nesse modo é possível visualizar e compreender como o trabalho realmente é executado através dos processos pelo rompimento das barreiras funcionais. Assim, é integrado aos processos o ambiente externo, representado, por exemplo, pelos clientes e fornecedores, os quais não integram o organograma tradicional (ver Figura 2).

Para Gonçalves (2000) existem vários estágios evolutivos na migração para uma estrutura orientada a processos, podendo ser identificados pelo menos cinco estágios, de um modelo puramente funcional até o essencialmente baseado em processos (Quadro 1).

Figura 2 - Estrutura funcional $X$ Estrutura por processos

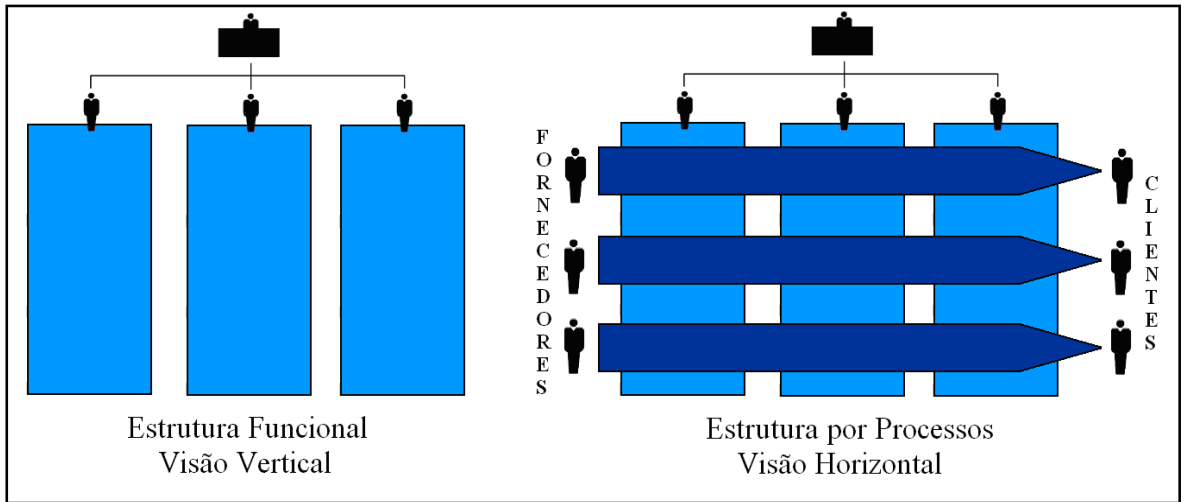

Fonte: Baseada em Rummler e Brache (1994)

Quadro 1 - Estágios para a gestão por processos

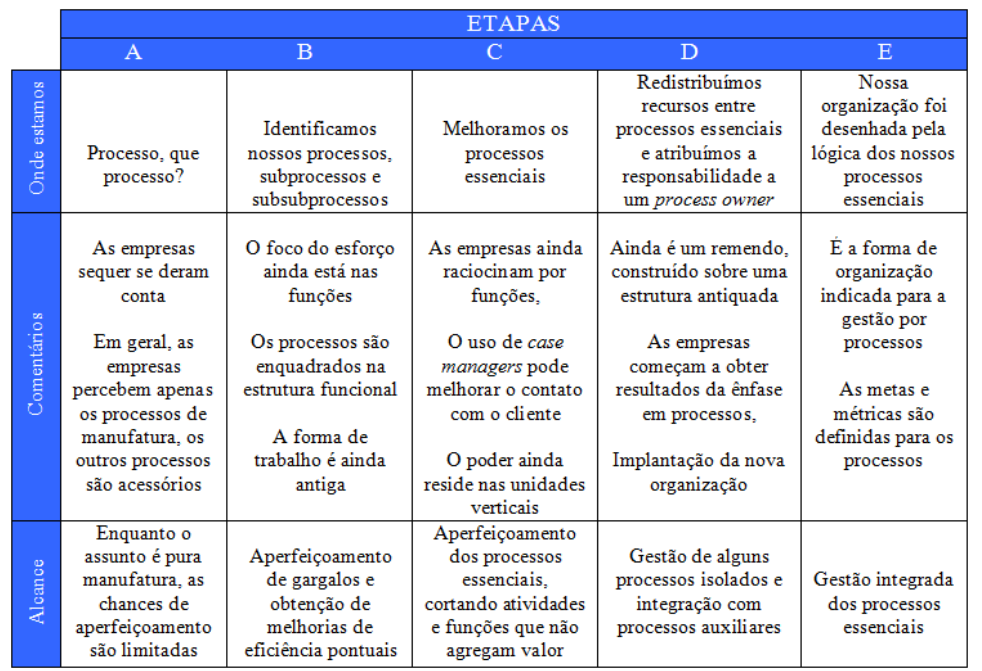

Fonte: Adaptado de Gonçalves (2000) 
Por fim, é coerente afirmar que essa transformação esteja sustentada por um método, para que a organização tenha possibilidade de conhecer os riscos envolvidos na migração e obtenha o melhor resultado. Do contrário, esse processo pode tender ao fracasso já nos primeiros passos (HAMMER, 1998; HAMMER; STANTON, 1999; VAN DER AALST et al., 2016).

\section{Comparativo de métodos de melhoramento e gestão de processos}

Objetivando encontrar o melhor caminho para a visão por processos, foi realizado um estudo sobre métodos que possibilitam introduzir esse conceito nas empresas. Foram analisadas as semelhanças entre os métodos encontrados e constatado que, essencialmente, a maioria destina-se a conhecer processos, identificar melhorias e garantir a implantação das melhorias. Porém, para um estudo mais detalhado, foram selecionados seis desses métodos pela proximidade de conceitos e etapas: o desenvolvido por Rummler-Brache (1994); o BPM de Elzinga et al. (1995); o apresentado por Pundek (2004); o método da UNICAMP (2003); o método TAD de Damij et al. (2008); e o método sintetizado por Almeida (2010).

No Quadro 2, são comparados esses seis métodos. Em uma primeira análise, observa-se que apresentam etapas muito semelhantes, que devem ser consideradas na implantação de cada um deles, porém, em um segundo momento, é perceptível que algumas fases são valorizadas na estruturação do método por alguns autores, por outros, não. Esse fato permite uma possível integração desses métodos partindo dessa análise comparativa possibilitando que, posteriormente, seja proposto um novo método.

Quadro 2 - Comparação dos métodos e identificação de seis fases da gestão por processos

\begin{tabular}{|c|c|c|c|c|c|c|}
\hline FASES & RUMMLER - BRACHE & BPM & PUNDEK & $\begin{array}{l}\text { GESTAOPOR } \\
\text { PROCESSOS- } \\
\text { UNICAMP } \\
\end{array}$ & TAD & ALMEIDA \\
\hline $\begin{array}{l}\text { CONHECIMENTO } \\
\text { DA OR GANIZAÇÃO }\end{array}$ & $\begin{array}{l}\text { 1) Identificação das } \\
\text { questões críticas do } \\
\text { negócio; }\end{array}$ & $\begin{array}{l}\text { 1) Preparação } \\
\text { para BPM; }\end{array}$ & & $\begin{array}{l}\text { 1) Planejamento } \\
\text { estratégico; } \\
\text { 2) Entendimento do } \\
\text { negócio; }\end{array}$ & & \\
\hline $\begin{array}{l}\text { SELEÇÃO DO } \\
\text { PROCESSO }\end{array}$ & $\begin{array}{l}\text { 2) Seleção dos processos } \\
\text { críticos; } \\
\text { 3) Seleção do líder e dos } \\
\text { membros da equipe; } \\
\text { 4) Treinamento da equipe; }\end{array}$ & $\begin{array}{l}\text { 2) Seleção do } \\
\text { processo; }\end{array}$ & $\begin{array}{l}\text { 1) Identificação dos processos- } \\
\text { chave da empresa; }\end{array}$ & $\begin{array}{l}\text { 3) Identificação dos } \\
\text { processos críticos; }\end{array}$ & $\begin{array}{l}\text { 1) Identificação } \\
\text { dos processos de } \\
\text { Negócio; }\end{array}$ & 1) Planejamento; \\
\hline $\begin{array}{l}\text { MAPEAMENTO DO } \\
\text { PROCESSO }\end{array}$ & $\begin{array}{l}\text { 5) Desenvolvimento do } \\
\text { mapa; }\end{array}$ & $\begin{array}{l}\text { 3) Descrição do } \\
\text { processo; }\end{array}$ & $\begin{array}{l}\text { 2) Estabelecimento, por processo, } \\
\text { das entradas, recursos e saídas; } \\
\text { 3) Análise e elaboração da matriz } \\
\text { de responsabilidades do processo; }\end{array}$ & $\begin{array}{l}\text { 4) Registros dos } \\
\text { clientes e } \\
\text { fomecedores do } \\
\text { processo; }\end{array}$ & $\begin{array}{l}\text { 2) Modelagem } \\
\text { dos processos de } \\
\text { negócio; }\end{array}$ & $\begin{array}{l}\text { 2) Mapeamento e } \\
\text { modelagem dos } \\
\text { processos; }\end{array}$ \\
\hline $\begin{array}{l}\text { ANÁLISE DO } \\
\text { PROCESSO }\end{array}$ & $\begin{array}{l}\text { 6) Identificação de } \\
\text { desconexões; } \\
\text { 7) Análise das } \\
\text { desconexões; } \\
\text { 8) Desenvolvimento do } \\
\text { mapa ideal; } \\
\text { 9) Estabelecimento de } \\
\text { medidas; }\end{array}$ & $\begin{array}{l}\text { 4) } \\
\text { Quantificação } \\
\text { do processo; } \\
\text { 5) Seleção de } \\
\text { melhorias; }\end{array}$ & 4) Estrutura ção das tarefas; & $\begin{array}{l}\text { 5) Análise do } \\
\text { processo atual; }\end{array}$ & $\begin{array}{l}\text { 3) Melhoria do } \\
\text { processo de } \\
\text { negócio; }\end{array}$ & \\
\hline $\begin{array}{l}\text { ESTABELECIMENTO } \\
\text { DE MUDANÇAS }\end{array}$ & $\begin{array}{l}\text { 10) Planejamento de } \\
\text { mudanças, } \\
\text { desenvolvimento de } \\
\text { recomendações e } \\
\text { apresentacãa; }\end{array}$ & & $\begin{array}{l}\text { 5) Elaboração do procedimento } \\
\text { operacional (PO); }\end{array}$ & $\begin{array}{l}\text { 6) Redesenho do } \\
\text { processo; }\end{array}$ & $\begin{array}{l}\text { 4) } \\
\text { Desenvolvimento } \\
\text { do modelo; } \\
\text { 5) Design; }\end{array}$ & $\begin{array}{l}\text { 3) Redesenho do } \\
\text { processo; } \\
\text { 4) Planejamento da } \\
\text { implantação/transição; }\end{array}$ \\
\hline $\begin{array}{l}\text { IMPLEMENTACÃO E } \\
\text { MONITORAMENTO } \\
\text { DO PROCESSO }\end{array}$ & $\begin{array}{l}\text { 11) implementação das } \\
\text { mudanças e } \\
\text { monitoramento; }\end{array}$ & $\begin{array}{c}\text { 6) } \\
\text { Implementação; }\end{array}$ & $\begin{array}{l}\text { 6) Implantação dos processos } \\
\text { padronizados; } \\
\text { 7) Monitoramento; }\end{array}$ & $\begin{array}{l}\text { 7) implantação do } \\
\text { processo; } \\
\text { 8) Gerenciamento do } \\
\text { processo; }\end{array}$ & $\begin{array}{c}\text { 6) } \\
\text { Implementação; }\end{array}$ & $\begin{array}{l}\text { 5) Capacitação das } \\
\text { pessoas envolvidas no } \\
\text { novo processo; } \\
\text { 6) Gestão da } \\
\text { transiçãa; }\end{array}$ \\
\hline
\end{tabular}

Fonte: Baseado em Almeida (2010); Campos et al. (2003); Damij et al. (2008); Elzinga et al. (1995); Pundek (2004); Rummler e Brache (1994) 
De maneira geral, a análise dos seis métodos permite identificar fases significativas e presentes em todos eles: conhecimento da organização; seleção do processo; mapeamento; análise; mudanças; implantação e monitoramento. Porém, cada método adota formas diferentes de abordagem dessas fases, como, por exemplo, identificação de rupturas e desenvolvimento do processo ideal (Rummler-Brache e Almeida); direta análise do desempenho dos processos (Gestão por Processos-UNICAMP); implantação de melhorias no processo já existente (Pundek, RummlerBrache e BPM); total redesenho (Gestão por Processos-UNICAMP, TAD e Almeida); essencial uso de software por uma (TAD) e por outras não (Gestão por Processos-UNICAMP, BPM, Pundek, Rummler-Brache e Almeida). Esse fato permite, portanto, uma integração de conceitos na busca do melhor resultado na introdução de um gerenciamento por processos, e a possibilidade de uma melhoria de processos mais eficaz.

Em suma, a gestão por processos deve seguir as fases definidas anteriormente. Todavia, primeiramente é importante conhecer a necessidade de melhoria. A partir disso, a escolha do método torna-se mais fácil e com mais chances de sucesso (Jacobs et al., 2009).

\section{Gerenciamento orientado a processos: proposta de um método}

Partindo da identificação das etapas relevantes para o gerenciamento de processos (Quadro 2), é proposto um método com base na integração daqueles já analisados. Pretende-se obter um conjunto de práticas mais adaptáveis às diversas situações nas quais se deseja implementar essa forma de gestão, pois os métodos mais eficientes para a gestão de processos não apresentam um conjunto ordenado de atividades e técnicas, mas um conjunto sugestivo que pode ser aplicado ou não (DE SORDI, 2008).

O método proposto será apresentado por meio de seis fases, com suas etapas e o produto final esperado de cada uma, como esquematizado no Quadro 3. 
Quadro 3 - Método de gerenciamento por processos

\begin{tabular}{|c|c|c|}
\hline \multicolumn{3}{|c|}{ METODO PROPOSTO } \\
\hline FASES & ETAPAS DE CADA FASE & PRODUT OS OBTIDOS \\
\hline $\begin{array}{c}\text { FASE I - } \\
\text { CONHECIMENTO } \\
\text { DA OR GANIZAÇÃO }\end{array}$ & $\begin{array}{l}\text { 1) Conhecimento do Planejamento Estratégico da } \\
\text { Organização, de sua missão, visão e metas, } \\
\text { 2) Identificação do funcionamento da organização } \\
\text { enquanto sistema pelo entendimento do negócio, } \\
\text { análise da cadeia de valores, mapeamento do } \\
\text { contexto organizacional e identificação dos } \\
\text { processos principais e de apoio da mesma; }\end{array}$ & $\begin{array}{l}\text { *uadro com a análise da } \\
\text { cadeia de valores da empresa } \\
\text { (entradas, processos e saidas); } \\
\text { * Mapa do contexto } \\
\text { organizacional, contendo uma } \\
\text { visão global dos } \\
\text { macroprocessos da empresa; }\end{array}$ \\
\hline $\begin{array}{l}\text { FASE II - SELEÇAO } \\
\text { DO PROCESSO }\end{array}$ & $\begin{array}{l}\text { 3) Seleção dos processos críticos do negócio; } \\
\text { 4) Seleção de representantes dos processos a serem } \\
\text { analisados; }\end{array}$ & $\begin{array}{l}* \text { Processos críticos } \\
\text { identificados; } \\
\text { * Representantes selecionados; }\end{array}$ \\
\hline $\begin{array}{l}\text { FASE III - } \\
\text { MAPEAMENTO DO } \\
\text { PROCESSO }\end{array}$ & $\begin{array}{l}\text { 5) Levantamento de todos os requisitos dos clientes } \\
\text { e dos fomecedores do processo a ser analisado; } \\
\text { 6) Descrição detalhada do processo a ser analisado } \\
\text { através da identificação das atividades e } \\
\text { subprocessos que compõem o macrofluxo de } \\
\text { trabalho; }\end{array}$ & $\begin{array}{l}\text { *Macrofluxo do processo } \\
\text { esquematizado; } \\
\text { * Subprocessos identificados; }\end{array}$ \\
\hline $\begin{array}{l}\text { FASE IV - ANÁLISE } \\
\text { DO PROCESSO }\end{array}$ & $\begin{array}{l}\text { 7) Elaboração do fluxograma geral do processo; } \\
\text { 8) Seleção das atividades (ou subprocessos) que } \\
\text { serão alvo de melhorias, através da identificação e } \\
\text { classificação, no processo estudado, de rupturas, } \\
\text { desconexões e pontos de impacto negativo; }\end{array}$ & $\begin{array}{l}\text { * Fluxograma geral do processo; } \\
\text { * Análise da situação atual do } \\
\text { processo; }\end{array}$ \\
\hline $\begin{array}{c}\text { FASE V- } \\
\text { ESTABELECIMENTO } \\
\text { DE MUDANÇAS }\end{array}$ & $\begin{array}{l}\text { 9) Estabelecimento de possiveis mudanças. Se } \\
\text { necessáno estabelecer um novo modelo para o } \\
\text { processo através de um redesenho do mesmo; } \\
\text { 10) Criação de um sistema de indicadores; }\end{array}$ & $\begin{array}{l}\text { * Sugestão de mudanças; } \\
\text { * Relatónio a ser entregue à } \\
\text { diretoria; } \\
\text { * Sistema de indicadores; }\end{array}$ \\
\hline $\begin{array}{l}\text { FASE VI - } \\
\text { IMPLEMENTAÇÃO E } \\
\text { MONITORAMENTO } \\
\text { DO PROCESSO }\end{array}$ & $\begin{array}{l}\text { 11) Criação dos procedimentos operacionais } \\
\text { padrão (POP's); } \\
\text { 12) Recomendação e implementação das melhorias } \\
\text { sobre o processo; } \\
\text { 13) Monitoramento e gerenciamento do processo; }\end{array}$ & $\begin{array}{l}\text { * POP`s elaborados; } \\
\text { * Inovações implantadas; } \\
\text { * Treinamento realizado; } \\
\text { * Sistema de gerenciamento do } \\
\text { processo; }\end{array}$ \\
\hline
\end{tabular}

\section{Fase I - Conhecimento da organização}

Esta fase baseia-se em Porter (1996) quando afirma que estratégia é a capacidade de uma empresa desempenhar atividades diferenciadas, mostrando a importância de haver um plano norteador na organização (MINTZBERG et al., 2009). O conhecimento dessa informação é, portanto, fundamental para compreender o que almeja a instituição. A mesma significância deve ser dada ao conhecimento do negócio. Para Porter (1989), toda empresa é um conjunto de atividades que projetam, produzem, comercializam, entregam e sustentam produtos e serviços e pode ser representada pela cadeia de valor. Por esse motivo, o conhecimento do negócio podese dar através da análise dessa cadeia de valor que permite identificar atividades de relevância estratégica.

\section{Fase II - Seleção do processo}

Esta fase inicia com a identificação, por meio de entrevistas com a gerência e análise do mapa do contexto, de rupturas ou pontos de impacto negativo. Essa identificação de falhas, ou fatores que merecem atenção por serem estratégicos, permite, de forma mais objetiva, a percepção dos processos que podem ser melhorados: os processos críticos (DE SORDI; MEDEIROS JUNIOR, 2006; BALDAM et al., 2007; ALBUQUERQUE; ROCHA, 2007). Por fim, 
deve haver uma seleção de representantes dos processos, pois esses indivíduos conhecem suas atividades, facilitando a análise, além de torná-la mais eficiente.

\section{Fase III - Mapeamento do processo}

Esta fase baseia-se em Harrington (1991), quando afirma que é através do mapeamento que se adquire conhecimento para aperfeiçoar processos. Com o desenvolvimento do mapa do processo obtém-se também, conforme Marrelli (2005) e Storch et al. (2013), uma visão sistêmica, pois se conhece melhor a inter-relação dos indivíduos no processo e as atividades que o compõem. Além disso, o autor comenta que é possível, por meio do mapeamento, conhecer os requisitos necessários para sua execução e as saídas esperadas.

\section{Fase IV - Análise do processo}

Esta fase abrange a análise do processo mapeado. Para isso, deve-se construir seu fluxograma, fonte de compreensão das etapas e atividades do processo e base para a tradução dos sintomas dos problemas (BALDAM et al., 2007). Em seguida, deve ocorrer a seleção das atividades (ou subprocessos) alvos de melhorias, pela identificação e classificação de rupturas e pontos de impacto negativo, com foco nas características esperadas, como frequência e modo de realização. A análise desses fatores pode ser feita através da criação de indicadores de desempenho (LADEIRA et al., 2012).

\section{Fase V - Estabelecimento de mudanças}

Esta fase compreende a definição do que é necessário alterar. Nesse caso podem ocorrer duas situações: melhorias nos processos já existentes (mudanças incrementais e tempo curto para realização), como apresentado nos métodos Rummler-Brache (1994), BPM (1995) e Pundek (2004); e reengenharia do processo (mudanças radicais, criação de um novo processo e tempo longo de estudo), conforme os métodos Gestão por Processos-UNICAMP (2003), TAD (2008) e Almeida (2010). É acrescido a essa etapa o desenvolvimento de um sistema de controle sobre as mudanças. Baldam et al. (2007), Albuquerque e Rocha (2007) e Ladeira et al. (2012) sugerem estruturar um sistema de indicadores no processo.

\section{Fase VI - Implementação e monitoramento do processo}

A última etapa compreende a implementação das mudanças e o gerenciamento do novo processo. Nessa fase, antecedendo a implementação, deve-se construir os novos POP's, que são importantes em qualquer processo, pois objetivam garantir que os resultados esperados sejam obtidos (COLENGHI, 1997). Com o cumprimento desta última fase, a instituição, a partir 
do gerenciamento dos processos críticos, introduz em seu contexto organizacional uma nova forma de gestão: o gerenciamento "por" processos.

\section{Método de pesquisa}

O presente estudo analisa o processo produtivo de uma empresa farmacêutica brasileira. É considerado um estudo de caso, pois implica uma análise de um fenômeno para criar um entendimento sobre ele (YIN, 2001). Para atingir seus objetivos, foram realizadas visitas in loco, onde os fatos foram observados, registrados, analisados, classificados e interpretados, sem interferência dos pesquisadores (ANDRADE, 2010). Também é característica desta pesquisa a preocupação em identificar fatores que determinam ou que contribuem para a ocorrência dos fenômenos, isto é, suas causas, o que, segundo Marconi e Lakatos (2017), caracteriza uma pesquisa explicativa. Desse modo, o trabalho é caracterizado como um estudo de caso exploratório explicativo.

\section{Estudo de caso}

A dedicação e o esforço das empresas para simplificar seus processos têm sido responsáveis pelo alcance de vantagens competitivas (BALDAM et al., 2007). Com as empresas do setor farmacêutico não é diferente, é crescente a busca por mudanças no seu modo de administrar, pois aspectos como custo, produtividade e otimização de tempo passaram a ser foco de estudos, o que permite, portanto, a introdução dos conceitos do modelo gerencial orientado a processos.

Por esse motivo, a definição do universo de pesquisa recaiu sobre uma farmácia de manipulação do Sul do Brasil que investe na busca da excelência em gestão. Será descrito o projeto de aplicação do método de gerenciamento por processos proposto neste trabalho, objetivando introduzir nessa organização conceitos deste modelo gerencial por meio da sugestão de melhorias para um de seus processos principais.

\section{Conhecimento da organização}

Antes de qualquer estudo relativo a processos, é necessário o entendimento da empresa como um sistema dinâmico. Para isso, deve-se verificar o que realmente agrega valor a seus produtos e serviços (ALBUQUERQUE; ROCHA, 2007). Foi analisada a cadeia de valor da empresa farmacêutica, apresentada na Figura 3, objetivando identificar seus processos principais e de apoio, bem como as entradas e saídas esperadas. 
Figura 3 - Cadeia de valor

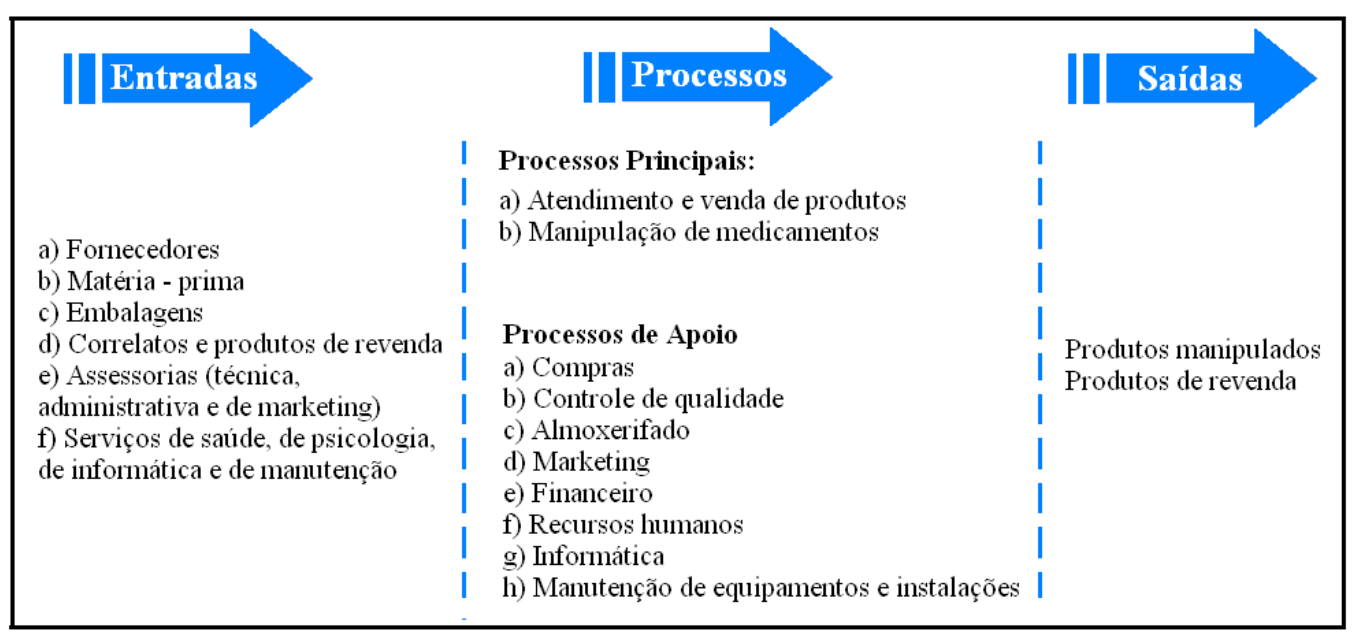

Em seguida, foi esquematizado o mapa do contexto organizacional que, segundo o método de Rummler e Brache (1994), possibilita entender a relação empresarial com seu ambiente de negócio. Através deste mapa, os macroprocessos puderam ser identificados por meio de uma visão horizontalizada possibilitando uma visualização das entradas e saídas que influenciam as funções e a identificação de falhas nos relacionamentos entre funções e departamentos.

\section{Seleção do processo}

Hammer (1998) afirma que mudar para uma visão por processos é difícil, pois exige uma nova forma de pensamento em que o negócio é compreendido claramente e os colaboradores assumem mais responsabilidades e trabalham mais em equipe. Por isso, optou-se pelo estudo de um dos processos principais, por possibilitarem resultados mais visíveis aos seus clientes externos e internos (DE SORDI, 2008).

Primeiramente foi feita a escolha de qual processo principal seria alvo do estudo, visto que existem dois (Figura 3). A decisão foi tomada tendo como referência a demanda por medicamentos manipulados. A pesquisa deteve-se, portanto, a uma análise sobre o processo de manipulação de medicamentos.

\section{Mapeamento do processo}

O próximo passo foi conhecer o processo escolhido mais profundamente e realizar um levantamento de dados acerca da sua situação atual, para que fosse possível identificar oportunidades de melhorias. Por essa razão, através da observação de campo, acompanhamento da produção, leitura dos POP's e entrevistas informais com colaboradores envolvidos na manipulação de medicamentos, foi possível construir o macrofluxo do processo (Figura 4). 
Figura 4 - Macrofluxo do processo

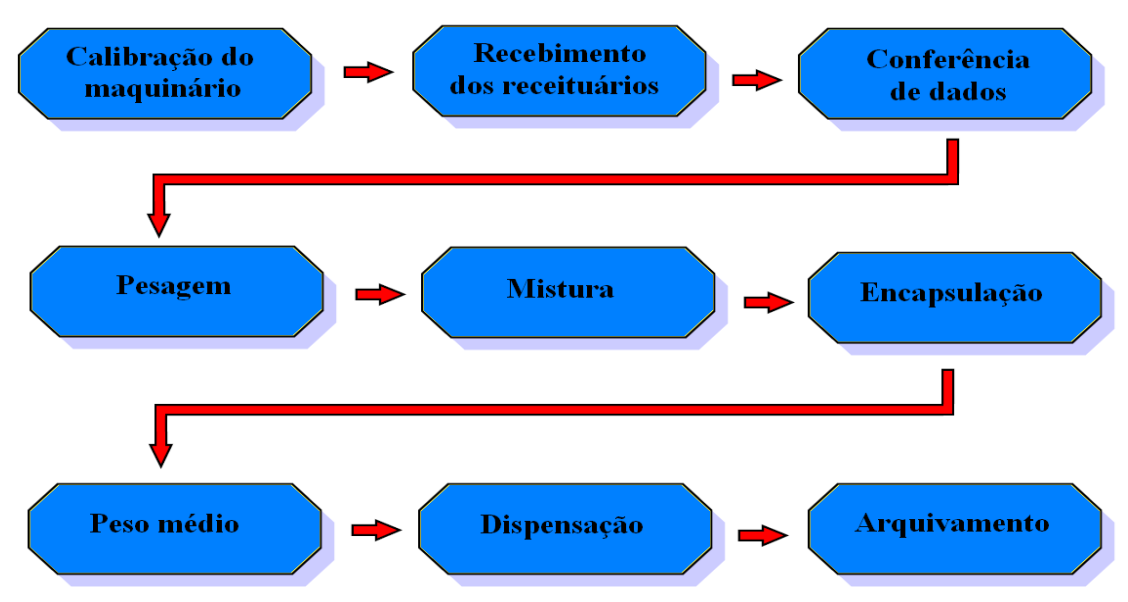

\section{Análise do processo}

Após construir o macrofluxo, o entendimento das atividades passou a ser primordial para se encontrar oportunidades de melhorias. Para isso, foi construído um fluxograma do processo que possibilitou a identificação de oportunidades de melhoria de diversas naturezas, ou rupturas, cujas localizações, dentro da cadeia produtiva, podem ser visualizadas em vermelho no próprio fluxograma, representado na Figura 5.

Quanto aos parâmetros de classificação, a equipe adotou a frequência com que o evento ocorre e a gravidade da ruptura: frequência alta (ocorre em mais de $80 \%$ das vezes em que a atividade é executada), média (entre $30 \%$ e $80 \%$ ) e baixa (menos de $30 \%$ ); a gravidade está relacionada ao efeito provocado pela ruptura em termos de perdas de tempo e prejuízo financeiro. As rupturas foram classificadas e representadas no Quadro $4 \mathrm{com}$ a legenda "A" para alta frequência ou alta gravidade; "M" para média e "B" para baixa. As rupturas com classificação "A/A" (alta frequência e alta gravidade) serão o foco do estudo. 
Figura 5 - Fluxograma e rupturas

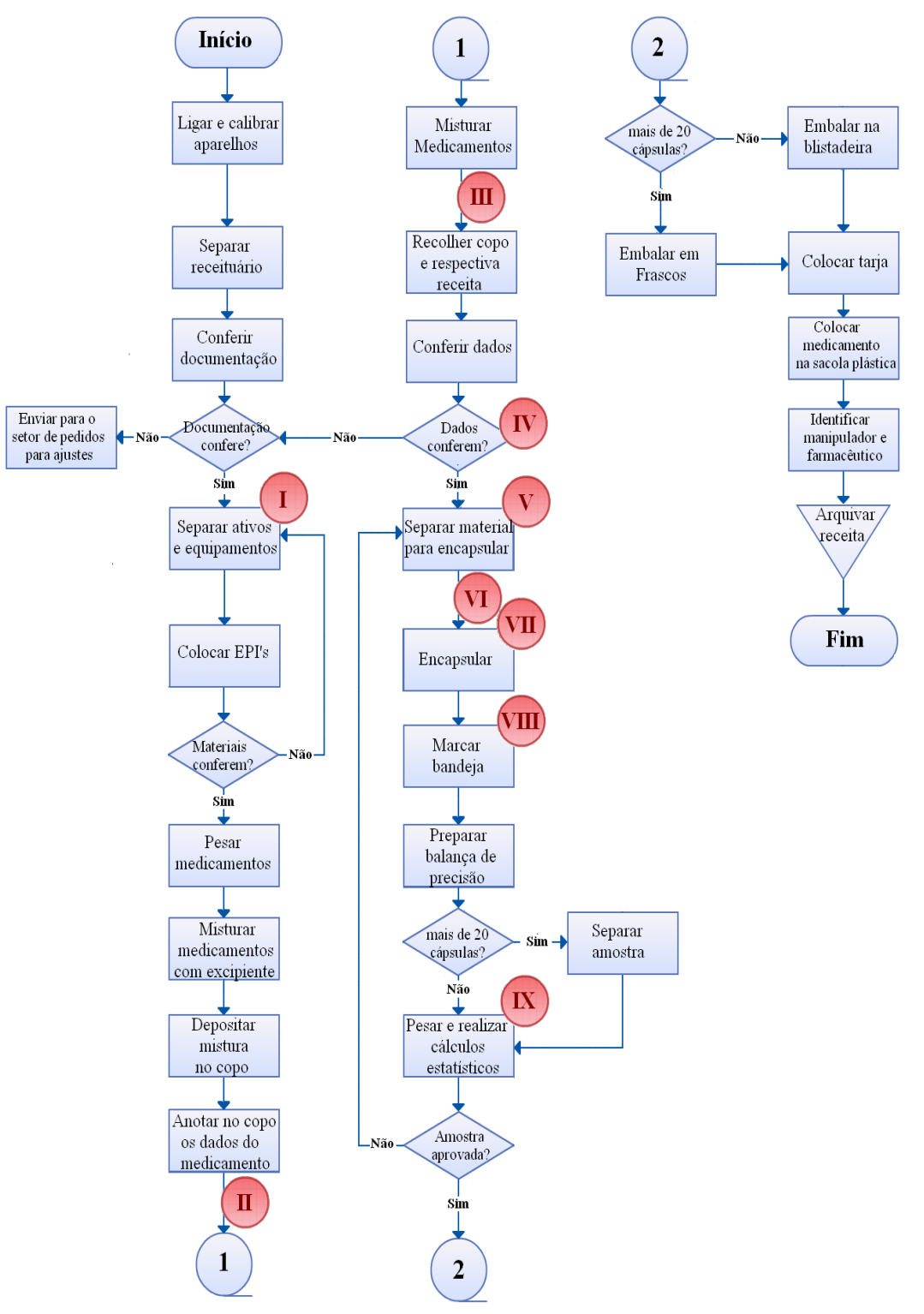




\begin{tabular}{|c|c|c|}
\hline Ruptura identificada & Impacto e frequência da ruptura & $\begin{array}{l}\text { Classificação para } \\
\text { gravidadeffrequência }\end{array}$ \\
\hline $\begin{array}{l}\text { I. \& V. Falta de layout } \\
\text { apropriado. Equipamentos } \\
\text { longe do local de uso }\end{array}$ & $\begin{array}{l}\text { Gera fluxo desnecessánio de pessoas dentro do } \\
\text { laboratónio. Isso atrapalha a eficiência da produção, } \\
\text { pois há gasto de tempo e estresse. }\end{array}$ & $\mathrm{M} / \mathrm{A}$ \\
\hline $\begin{array}{l}\text { II. Medicamentos esperando } \\
\text { misturador ocupa do }\end{array}$ & $\begin{array}{l}\text { O impacto está relacionado à diminuição da } \\
\text { eficiência e da produtividade, representando, } \\
\text { portanto, um impacto negativo. Ocorre quase que ma } \\
\text { totalidade da produção, dependendo da demanda. }\end{array}$ & $\mathrm{A} / \mathrm{A}$ \\
\hline $\begin{array}{l}\text { III. Demora na mistura } \\
\underline{\text { manual dos medicamentos }}\end{array}$ & $\begin{array}{l}\text { O impacto está relacionado à diminuição da } \\
\text { eficiência do processo da produtividade, pois além da } \\
\text { demora é realizado manualmente e o responsável tem } \\
\text { seu rendimento reduzido e, portanto, um impacto } \\
\text { negativo. Ocorre na totalidade da produção. }\end{array}$ & $\mathrm{A} / \mathrm{A}$ \\
\hline $\begin{array}{l}\text { IV. Falta de controle mais } \\
\text { eficiente sobre o receituánio }\end{array}$ & $\begin{array}{l}\text { O impacto está relacionado à necessidade de refazer } \\
\text { todo o procedimento, gerando, assim, gastos } \\
\text { desnecessános e tempo perdido. Sua frequência é } \\
\text { raríssima, haja vista a experiência dos colaboradores. }\end{array}$ & $\mathrm{A} / \mathrm{B}$ \\
\hline $\begin{array}{l}\text { VI. Perda de medicamento no } \\
\text { copo plástico }\end{array}$ & $\begin{array}{l}\text { Os impactos estão relacionados à perda de matéria e, } \\
\text { consequentemente, gastos desnecessánios. Ocorre } \\
\text { com todos os medicamentos produzidos. }\end{array}$ & $\mathrm{M} / \mathrm{A}$ \\
\hline $\begin{array}{l}\text { VII. Demora do processo de } \\
\text { encapsulação que é } \\
\text { totalmente manual }\end{array}$ & $\begin{array}{l}\text { Diminuição da produtividade e eficiência, haja vista o } \\
\text { processo de encapsulação ser manual. Ocorre durante } \\
\text { toda a produção, prejudicando apenas quando a } \\
\text { demanda é máxima. }\end{array}$ & $\mathrm{B} / \mathrm{M}$ \\
\hline $\begin{array}{l}\text { VIII. Falta de um melhor } \\
\text { controle sobre as marcações } \\
\text { das partes usadas da bandeja. }\end{array}$ & $\begin{array}{l}\text { Impacto sobre o entendimento das partes utilizadas, } \\
\text { prejudicando a eficiência da produção. Ocorre para } \\
\text { todas as bandejas utilizadas na encapsulação. }\end{array}$ & $\mathrm{M} / \mathrm{A}$ \\
\hline $\begin{array}{l}\text { IX. Cálculo manual do peso } \\
\text { médio e coeficiente de } \\
\text { variação das cápsulas }\end{array}$ & $\begin{array}{l}\text { Tempo de cálculo grande, impedindo que } 0 \\
\text { colaborador dedique mais tempo à produção. Além } \\
\text { disso, por ser manual, reduz a confiabilidade sobre } \circ \\
\text { medicamento produzido e o controle sobre a } \\
\text { produção. O problema ocorre em todo o processo de } \\
\text { forma contínua. }\end{array}$ & $\mathrm{A} / \mathrm{A}$ \\
\hline
\end{tabular}

\section{Estabelecimento de mudanças}

Em seguida foi possível estruturar um plano de ação que permitisse a sugestão de melhorias que solucionassem as principais rupturas. Para isso, foi efetuado um estudo de um mês na produção (21 dias úteis). Nesse período foram feitas medições dos tempos de cada processo.

A partir dessa análise, os subprocessos que mais exigiam dedicação dos colaboradores foram: "mistura" e "peso médio" (Figura 6). A principal razão para que esses subprocessos fossem considerados gargalos é o impedimento de os colaboradores estarem disponíveis para a execução de outras tarefas que agreguem mais valor ao processo.

Figura 6 - Tempos dos subprocessos

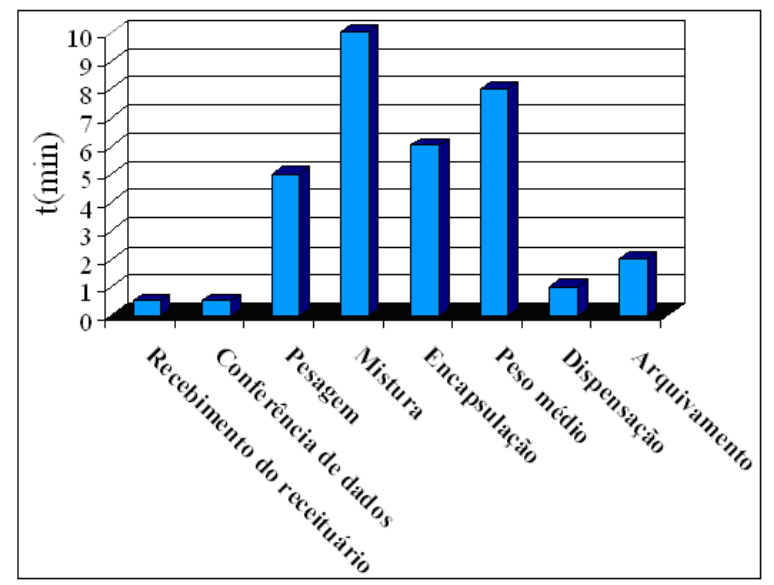


A mistura dos componentes é considerada um gargalo, atrasando o restante da produção, visto que não há capacidade para atender à demanda de medicamentos já pesados, acumulados neste ponto do processo. Para que a ruptura seja eliminada e a produção otimizada foi sugerida a utilização de um misturador automático, capaz de reduzir $9 \%$ do tempo do processo, além de possibilitar a eliminação do trabalho manual do colaborador, que pode ser melhor alocado no processo, aumentando a eficiência e a produtividade.

O cálculo do peso médio também é considerado um gargalo, pois o tempo gasto é significativo: $24 \%$ do tempo total. Além disso, impede que o colaborador se dedique a atividades que agreguem valor ao produto. Por ser realizado manualmente, reduz a confiabilidade sobre o medicamento e o controle sobre a produção. Para que essa ruptura tenha seu impacto reduzido foi sugerida a compra de um processador estatístico. Dessa forma o processo terá redução de $21 \%$ no tempo, eliminando o trabalho manual do colaborador que pode ser mais bem alocado, melhorando, também, a eficiência e a produtividade.

Caso as duas sugestões forem aceitas, o tempo de produção de uma unidade (33 minutos) reduzirá aproximadamente $30 \%, 3$ minutos pelo misturador automático e 7 minutos pelo processador estatístico, passando para 23 minutos, como sintetizado no Quadro 5. A mesma análise pode ser realizada para o tempo de ciclo do produto (compreendido como a divisão do tempo total da unidade pelas estações). Atualmente, a capacidade de produção está em 16 medicamentos por hora e possui oito estações de trabalho, com isso, o tempo de ciclo corresponde a 3,17 minutos. Com a adoção das duas sugestões e mantendo as oito estações de trabalho, a capacidade produtiva passará para 21 medicamentos por hora e, consequentemente, o tempo de ciclo para 2,88 minutos, correspondendo a uma redução de $23 \%$ do tempo e aumento de $31 \%$ da capacidade produtiva, como sintetizado no Quadro 5.

Quadro 5 - Síntese das mudanças

\begin{tabular}{|c|c|c|c|}
\hline Indice & Processo Atual & $\begin{array}{c}\text { Com as Mudanças } \\
\text { Sugeridas }\end{array}$ & $\begin{array}{c}\text { Quantificação da } \\
\text { melhoria }\end{array}$ \\
\hline $\begin{array}{c}\text { Tempo total gasto para a } \\
\text { produção de uma unida de }\end{array}$ & $33 \mathrm{~min}$ & $23 \mathrm{~min}$ & $-30 \%$ \\
\hline Tempo de ciclo & $3,75 \mathrm{~min}$ & $2,88 \mathrm{~min}$ & $-23 \%$ \\
\hline Capacidade produtiva & 16 medicamentos $\mathrm{h}$ & 21 medicamentos/h & $+31 \%$ \\
\hline
\end{tabular}

Outra sugestão realizada à empresa, foi a adoção de alguns indicadores para que haja um monitoramento caso as mudanças sejam adotadas no processo. Esses indicadores visam um feedback sobre o desempenho das alterações e a identificação de necessidades de novas mudanças, como sugerido pelo método proposto neste trabalho (BALDAM et al., 2007; ALBUQUERQUE; ROCHA, 2007; LADEIRA et al., 2012). Pelo uso de indicadores para o monitoramento do processo, a empresa poderá mensurar e quantificar o desempenho de seu processo e compreender o quão próximo está do esperado com as mudanças. Foram, então, sugeridos cinco indicadores, apresentados no Quadro 6, fundamentados nos dados disponíveis 
pelo processo: tempo de execução das atividades, número de manutenções, número de produtos conformes.

\begin{tabular}{|c|c|c|}
\hline $\begin{array}{l}\text { Classificação do } \\
\text { Indica dor }\end{array}$ & Indica dor & Finalidade \\
\hline $\begin{array}{l}\text { Indicadores de } \\
\text { qualidade (IQ) }\end{array}$ & Conformidades $=\frac{\text { Total de Medicamento Conformes } \times 100}{\text { Total de Medicamento Produzidos }}$ & $\begin{array}{l}\text { Identificar } \circ \text { percentual de } \\
\text { conformidade nos produtos } \\
\text { produzidos. }\end{array}$ \\
\hline \multirow{2}{*}{$\begin{array}{l}\text { Indicadores de } \\
\text { produtividade } \\
\text { (IP) }\end{array}$} & Atrasos na Entrega $=\frac{\text { Atrasos na Entrega }}{\text { Total de Medicamentos Produzidos }}$ & $\begin{array}{l}\text { Identificar o número de atrasos } \\
\text { nas entregas dos medicamentos } \\
\text { produzidos. }\end{array}$ \\
\hline & Tempo de Ciclo $=\frac{\text { Tempo de Produção }}{\text { Número de Pedidos }}$ & $\begin{array}{l}\text { Identificar o tempo ciclo do } \\
\text { medicamento no processo de } \\
\text { produção. }\end{array}$ \\
\hline \multirow{2}{*}{$\begin{array}{l}\text { Indicadores de } \\
\text { capacidade (IC) }\end{array}$} & Ociosidade $=\frac{\text { Tempo Disponivel dos Colaboradores }}{\text { Tempo Total de Produçäo }}$ & $\begin{array}{l}\text { Identificar o tempo ocioso dos } \\
\text { funcionánios durante a } \\
\text { produção. }\end{array}$ \\
\hline & Manutenção $=\frac{\text { Manutenções }}{\text { Tempo de Produção }}$ & $\begin{array}{l}\text { Identificar } \circ \text { número de } \\
\text { manutenções dos equipamentos. }\end{array}$ \\
\hline
\end{tabular}

Vale ressaltar que o método proposto visa a sugestão de indicadores que possibilitem futuras análises do processo. Dessa forma, a empresa poderá monitorar as mudanças que realizar e manter viva a essência da gestão por processos, pois terá maior controle sobre as alterações, além de dados para que um novo ciclo de análise e investigação se estabeleça.

\section{Implementação e monitoramento do processo}

A aplicação do método proposto neste estudo limitou-se à sugestão das mudanças no processo através de um relatório entregue à empresa contendo a análise do processo atual, sugestões de melhorias e seus benefícios, não acompanhando a decisão gerencial e implementação dessas mudanças. Porém, é fundamental destacar alguns aspectos sobre a implementação.

O sucesso da implementação está relacionado com as pessoas que irão executá-la e, por isso, deve-se mantê-las ligadas ao processo de transformação. Nesse sentido, a escolha dos colaboradores para auxiliar na obtenção de informações sobre o processo, como sugerido no método, possui um sentido maior: o de entrosamento e conhecimento por elas da proposta de um gerenciamento orientado a processos e a motivação para que sejam responsáveis pela implementação em seus setores através das melhorias nos processos.

Dessa forma, torna-se fundamental a elaboração de um plano para a renovação da cultura organizacional, passo essencial para uma bem-sucedida implementação da gestão por processos. Porém, o mais importante é que tal processo de renovação inicie nas primeiras fases do projeto, com o estudo do contexto e construção do mapa, e deve ter, com o decorrer das atividades, um tratamento sistemático e concentrado na fase de transição. 


\section{Considerações finais}

Considerando as novas necessidades das organizações, resultantes da atual conjuntura de mercado, que exige reestruturação dos processos para que os objetivos estratégicos sejam atingidos, este trabalho fornece subsídios para que um planejamento voltado a essa temática seja estruturado. Isso se dá por dois motivos: pela proposição de um modelo que possibilita a introdução de conceitos sobre o gerenciamento por processo na organização e por uma aplicação prática na qual se pôde analisar um processo de forma a testar o método proposto e sua capacidade de identificação de gargalos e a sugestão de alterações visando sua melhoria.

Com base nesses resultados, acredita-se que:

a) O modelo proposto é mais abrangente que os analisados e permite obter resultados válidos, pois, no estudo de caso, possibilitou um aumento do controle produtivo por indicadores, uma redução de $23 \%$ no tempo de ciclo e de $30 \%$ no tempo gasto na produção unitária, resultando em um aumento de produtividade de $31 \%$ no processo analisado;

b) Embora o método tenha foco na gestão de processos, é um instrumento que beneficia a organização na transição de uma visão tradicional para uma visão horizontalizada, pela inclusão dos colaboradores no projeto, possibilitando mudanças no pensamento organizacional da empresa.

Conforme observado, desde o início do estudo e implantação do método de gestão por processos, houve evolução organizacional, observada pelo aumento do conhecimento por parte da empresa sobre essa nova forma de gerenciamento, pela conscientização dos colaboradores e da alta direção sobre a necessidade dessa mudança, e pelo empenho de todos na busca de informações para a tomada de decisão, fatores essenciais para a consolidação da gestão por processos. Por isso, a pesquisa para o desenvolvimento deste trabalho foi importante para a organização, haja vista ter sido responsável pela introdução dos conceitos sobre esse modelo de gerenciamento no contexto da empresa.

Por fim, o presente estudo apresenta algumas limitações e possibilidades de estudos futuros. Um deles diz respeito ao acompanhamento da implantação das mudanças e indicadores sugeridos, cuja realização não foi abordada nessa pesquisa, visto que objetivava somente a utilização do método para análise e sugestão de melhorias em um processo da empresa. Dessa forma estudos futuros poderiam realizar a implementação da totalidade das fases do método proposto, bem como a realização de um novo ciclo de análise sobre o processo modificado e a adoção do método por outros processos da empresa. Assim, indícios serão obtidos sobre avanços organizacionais em direção a uma cultura e visão mais horizontalizada.

\section{Referências}

ALBUQUERQUE, A.; ROCHA, P. Sincronismo organizacional: como alinhar a estratégia, os processos e as pessoas. São Paulo: Saraiva, 2007.

ALMEIDA, L.L.P. A gestão orientada a processos aplicada a uma indústria da moda, 2010. (Dissertação de Mestrado) - Faculdade de Economia e Finanças IBMEC, Rio de Janeiro, 2010. 
ANDRADE, M.M. Introdução à metodologia do trabalho científico. 10. ed. São Paulo: Atlas, 2010.

ARAÚJO, L.C.G. Organização, sistemas e métodos e as tecnologias de gestão organizacional. 4. ed. São Paulo: Atlas, 2012.

BALDAM, R. et al. Gerenciamento de processos de negócio: BPM business process management 2. ed. São Paulo: Érica, 2007.

CAMPOS, E. R. Metodologia de Gestão por processos. Campinas: UNICAMP, 2003. Disponível em: < http://www.prdu.unicamp.br/gestao_por_processos/gestao_processos.html > Acesso em: 27 de nov. 2017.

COLENGHI, V. M. O\&M e qualidade total: uma integração perfeita. Rio de Janeiro: Qualitymark, 1997.

DAMIJ, N.; DAMIJ, T.; GRAD, J.; JELENC, F. A methodology for business process improvement and IS development. Information and Software Technology, v. 50, n. 11, p. 1127-1141, 2008.

DAVENPORT, T.H. Process innovation: reengineering work through information technology. Boston: Harvard Business School Press, 1993.

DE SORDI, J.O. Gestão por processos: uma abordagem da moderna administração. 2. ed. São Paulo: Saraiva, 2008.

DE SORDI, J.O.; MEDEIROS JÚNIOR, G. Abordagem sistêmica para integração entre sistemas de informação e sua importância à gestão da operação: análise do caso GVT. Gestão \& Produção, v. 13, n. 1, p. 105-116, 2006.

ELZINGA, D.J.; HORAK, T.; LEE, C.Y.; BRUNER, C. Business process management: survey and methodology. IEEE Transactions on Engineering Management, v. 42, n. 2, p. 119-128, 1995.

GONÇALVES, J.E.L. Processo, que processo? RAE - Revista de Administração de Empresas, v. 40, n. 4, 2000.

GUHA, S.; KETTINGER, W.J. Business process reengineering: building a comprehensive methodology. Information Systems Management, v. 10, n. 3, p. 13-22, 1993.

HAMMER, M.A. Empresa voltada para processos. Revista HSM Management, n. 9, ano 2, 1998.

HAMMER, M.; STANTON, S. How process enterprises really work. Harvard Business Review, v. 77 , n. 6, p. 108-118, 1999.

HARRINGTON, H. J. Business process improvement. New York: McGraw Hill, 1991.

IDEN, J. Investigating process management in firms with quality systems: a multi-case study. Business Process Management Journal, v.18, n. 1, p. 104-121, 2012.

JACOBS, G.; KIPPER, L.M.; ELLWANGER, M.C.; FROZZA, R.; NARA, E.O.B. Por onde começar a gestão por processos? uma análise sobre metodologias. In: Anais do XVI Simpósio de Engenharia de Produção, Botucatu: UNESP, 2009.

LADEIRA, M.B.; RESENDE, P.T.V.; OLIVEIRA, M.P.V.; MC CORMACK, K.; SOUSA, P.R.; FERREIRA, R.L. Gestão de processos, indicadores analíticos e impactos dobre o desempenho competitivo em grandes e médias empresas brasileiras dos setores da indústria e serviços. Gestão \& Produção, v. 19, n.2, p. 389-404, 2012.

MARANHÃO, M.; MACIEIRA, M.E.B. O Processo nosso de cada dia: modelagem de processos de trabalho. Rio de Janeiro: Qualitymark, 2004. 
MARCONI, M.A; LAKATOS, E.M. Fundamentos de metodologia científica. 8. ed. São Paulo: Atlas, 2017.

MARRELLI, A.F. The performance technologist's toolbox process mapping. Performance Improvement. v. 44 n. 5, p. 40-44, 2005.

MINTZBERG, H.; AHLSTRAND, B.; LAMPEL, J. Strategy safari: the complete guide through the wilds of strategic management. São Paulo: Pearson Prentice Hall, 2009.

PORTER, M.E. Vantagem competitiva: criando e sustentando um desempenho superior. Rio de Janeiro: Campus, 1989.

PORTER, M. E. What is strategy? Harvard Business Review, nov./dez, p. 61-78, 1996.

RECKER, J.; MENDLING, J. The State of the Art of Business Process Management Research as Published in the BPM Conference. Bus Inf Syst Eng v. 58, n. 1, p. 55-72, 2016.

RUMMLER, G.A.; BRACHE, A.P. Melhores desempenhos das empresas: uma abordagem prática para transformar a organização através da reengenharia. 2. ed. São Paulo: Makron Books, 1994.

PUNDEK, G.N.B. Metodologia para implantação conjunta de gestão orientada a processo e ERP. 2004. (Dissertação de Mestrado) - Pontifícia Universidade Católica do Paraná, Curitiba, 2004.

SLACK, N.; CHAMBERS, S.; JOHNSTON, R.; BETTS, A. Gerenciamento de operações e de processos: princípios e práticas de impacto estratégico. Porto Alegre: Bookman, 2008.

STORCH, L.A.; NARA, E.O.B.; KIPPER, L.M. The use of process management based on a systemic approach. International Journal of Productivity and Performance Management, v. 62, n. 7, 2013.

TRKMAN, P. The critical success factors of business process management. International Journal of Information Management, v. 30, n. 2, p. 125-134, 2010.

VAN DER AALST, W.M.P.; LA ROSA, M.; SANTORO, F.M. Business Process Management: Don't Forget to Improve the Process! Bus Inf Syst Eng, v. 58, n. 1, p. 1-6, 2016.

YIN, R.K. Estudo de caso: planejamento e métodos. 2. ed. Porto Alegre: Bookman, 2001. 